\title{
Transdisciplina y multidimensionalidad: una visión desde el contexto educativo
}

\author{
Suaza Jiménez, Jorge Hernán \\ Instituto Tecnológico Metropolitano, Colombia \\ jorgesuaza@itm.edu.co
}

Resumen - Las nuevas formas de pensamiento dan lugar a ciencias que se dedican a su comprensión. El pensamiento complejo y los problemas como sistemas complejos se pueden observar desde el punto de vista de lo multidimensional y desde la necesidad de incorporar múltiples disciplinas para hacerles frente. Esta no es una excepción en el contexto educativo, donde los estudiantes tienen múltiples formas y estilos de aprendizaje y se tiene el propósito de mejorar la forma en que se comprende el mundo de forma sistemática. Aun así, muchas instituciones no están preparadas para ello. Por ende, se definen los conceptos de transdisciplina y multidimensionalidad y se analiza, en cada uno, su importancia en la solución de problemas reales desde la teoría de la complejidad.

\section{Palabras clave - $\quad$ Transdisciplina;} Multidimensionalidad; Educación; Resolución de problemas; Pensamiento complejo;

\begin{abstract}
The new ways of thinking lead to sciences that are dedicated to their understanding. Complex thinking and the problems as complex systems can be seen from the point of view of multidimensionality and from the need of incorporate multiple disciplines to face them. This is not an exception in the educative context where the students have multiple ways and learning styles and the purpose is to improve the way the world is understood systematically. Still, many institutions are not prepared for it. Therefore, the concepts of transdiscipline and multidimensionality are defined
\end{abstract}

Interconectando Saberes, 2020

ISSN: 2448-8704 and their importance in the solution of real problems from the theory of complexity is analyzed on each one.

Keywords - Transdiscipline; Multidimensionality; Education; Problem solving; Complex thinking;

\section{INTRODUCCIÓN}

La educación contemporánea se enfrenta a nuevos retos, entre los que se encuentran el incremento acelerado de la información y la incertidumbre social y de los conocimientos. Para atender desafíos como estos, los profesionales no se pueden enmarcar en un solo nivel o dimensión de complejidad. Se hace necesaria una abstracción de estos, por medio de la lógica, para estructurar soluciones eficaces y eficientes. La transdisciplinariedad permite abordar estos problemas de forma sistemática. Mientras la disciplina se enfrenta a un solo nivel de realidad o dimensión, la transdisciplina atraviesa las barreras de estas dimensiones y pasa a ser multidimensional que permite establecer alternativas (múltiples dimensiones) para que las

Fecha de Recepción: 11 de mayo de 2020

Fecha de Aceptación: 07 de julio de 2020

Fecha de Publicación: 24 de julio de 2020 
sociedades actúen frente a estas situaciones problemáticas y su actividad continúe en el tiempo.

Esta sociedad moderna necesita creadores de soluciones que respondan a las exigencias del medio con ideas creativas e innovadoras, que no se limiten a memorizar el conocimiento ni a ejecutar tareas. Para llevar esto a cabo, se necesita incorporar en los procesos formativos tanto la lógica como la abstracción y la creatividad

De acuerdo con Maldonado (2007), este cambio de paradigma no solo implica abandonar modelo lineal, positivista y vectorial que ha regido desde la época de Descartes y Platón y que trajo consigo grandes progresos del conocimiento científico, sino volver a definir el concepto de ciencia ya que, aquello que caracteriza a la complejidad es el rompimiento de las comprensiones monolíticas y disciplinares del conocimiento, de modo que se puedan abarcar y proponer enfoques interdisciplinarios y transversales.

Aunque parezca distante, aún se educa a los estudiantes como en la era industrial, donde la meta eran las fábricas y las líneas de producción. El propósito de la educación, en ese entonces, era formar obreros competentes en áreas específicas, pero sin conocimientos muy profundos. "En esas instituciones educativas se tomaba a una persona y se le entrenaba, y cuando alcanzaba cierto grado de diferenciación con los demás se le consideraba competente, por lo que era certificada para ir a trabajar en la fábrica. Este concepto todavía sobrevive en el sistema actual" (Serna 2015)
La base de la enseñanza tradicional es, entonces, el aprendizaje de memoria, el seguimiento de planes de estudio rígidos, de formas de evaluación y contenidos obsoletos, que el sistema soporta y que se usan para verificar que el estudiante sea apto para cumplir con los estándares establecidos para todos.

Actualmente, este modelo de enseñanza no está funcionando porque, con el paso del tiempo, se producen cambios a nivel social científico y, aún más rápido, a nivel tecnológico.

Los nativos digitales y la sociedad actual tienen necesidades y ritmos diferentes a los de hace cien años. Ya no requieren un sistema de educación sino uno de formación que les permita crecer como personas, desarrollar habilidades y capacidades tales como la creatividad y la innovación para que sean profesionales que le aporten un valor agregado, un factor diferenciador al mercado laboral y al mundo, ya que, al contrario de la era industrial, donde se valoraba el estándar, ahora se aprecia la unicidad del individuo, su iniciativa y capacidad de tomar decisiones.

El proceso de enseñanza-aprendizaje deberá entonces tomar ventaja de las transformaciones de esta era a través del entendimiento del problema y su disgregación en elementos funcionales interrelacionados a través de una formación multidisciplinar y transdisciplinar donde los profesionales puedan enfrentar los retos planteando y buscando soluciones a problemas de forma eficiente; con una visión total, pero sin perder 
de vista lo parcial; con pensamientos abiertos y flexibles; con la capacidad de interacción con su ambiente, el entorno y con otros seres; responder y aprender a lo que sucede a su alrededor y poseer la capacidad de realizar actividades de acuerdo con sus motivaciones. Todo esto, apoyados en el uso de tecnologías de la información y la comunicación (TIC) donde se pueda gestionar el conocimiento de forma colaborativa.

\section{TRANSDISCIPLINA}

Según Nicolescu (1996), el objetivo de la transdisciplinariedad es comprender el mundo real, ya que representa la unidad del conocimiento. La transdisciplinariedad hace referencia a todas las cosas que tienen en común las diferentes disciplinas. Es una visión general y global que permite la discusión de temas tales como la educación, la ética, la sociedad y la ciencia.

A finales del siglo $X X$ hubo dos corrientes centradas en la transdisciplinariedad que capturaron bastante atención: la primera es la del ya mencionado Basarab Nicolescu (1996), cuya perspectiva de la transdisciplinariedad tenía un enfoque científico y cultural que facilitaba el diálogo entre especialistas de la ciencia quienes tenían una visión compleja de ese mundo.

Para Nicolescu, la transdisciplinariedad es un arte y una ciencia que permite el descubrimiento de puentes entre diferentes áreas del conocimiento y diferentes seres. Su principal labor es la construcción de un lenguaje lógico y la estructuración de los conceptos para lograr un diálogo real. Es una visión que comprende la ética, la creatividad, la transnacionalidad y la transculturalidad, y reconoce la multidimiensionalidad.

La segunda corriente se enfoca en los aspectos investigativos y de resolución de problemas a partir de la transdisciplinariedad, la complejidad y la transectorialidad como un conjunto de problemas que no nacen de la ciencia (Häberli et al., 2001). Desde este punto de vista, los problemas de una sociedad, al ser cada vez más complejos e interdependientes, no pueden aislarse en un sector o disciplina en particular y son impredecibles. La necesidad de la transdisciplinariedad es omnipresente ya que los problemas tienen características tales como la no-linealidad, la incertidumbre, los dominios complejos y múltiples, y las grandes apuestas políticas en la toma de decisiones (Goorhuis, 2000). Dichos problemas surgen tanto en campos donde los seres humanos interactúan con la naturaleza (agricultura, silvicultura, industria, entre otros) como en aquellos campos relacionados con los desarrollos tecnológicos (nucleares, biotecnológicos, genéticos), sociales, económicos y culturales (Häberli et al., 2001a). Los temas abarcados en cada uno de estos campos fueron concebidos, en un principio, para limitarse a su respectiva disciplina o sector, pero se ha comprendido su multidimensionalidad y la importancia de comprender y eliminar los límites de un pensamiento que se segmenta en función de solucionar los problemas. 
Para Nicolescu (1996) la transdisciplinariedad está basada en tres pilares esenciales: los niveles de la realidad, el principio del tercero incluido y la complejidad. La evolución ha mostrado que a partir de la comunicación oral donde, por medio de mitos e historias, el conocimiento pasa a la comunicación escrita, el pensamiento racional ha primado sobre el relacional, nublando otras capacidades y sentimientos que permitían comprender la naturaleza desde el interior. Por el contrario, según el autor, tanto lo racional como lo relacional son modos simultáneos de razonamiento. Los aspectos o pilares mencionados, que para Nicolescu son fundamentales, permiten comprender de forma pragmática estos modos de pensamiento.

En cuanto los niveles de la realidad, el primer pilar, la realidad es aquello que se resiste a toda experiencia, representación, imagen, descripción o formalización matemática. Por ejemplo, en la física cuántica, donde la abstracción describe una de las partes constitutivas de la naturaleza, en lugar de "la realidad", se demuestra que es imposible separar la formalización matemática de la experiencia (Nicolescu, 2000). A pesar de la búsqueda en el universo interior y la coexistencia de ambos mundos, hace falta reconocer que, aunque los estudios de transdisciplina son necesarios, esta per se es un concepto incompleto sobre el que hace falta mucho por descubrir. En este punto, la transdisciplinariedad funciona como una herramienta, tanto como un proyecto.
En el principio del tercero incluido, se comprende la transdisciplinariedad y la complejidad como una forma transversal y coherente de recorrer de forma iterativa las diferentes áreas del conocimiento con el propósito de lograr una simplicidad. Es complementario a la lógica del tercero excluido. Nicolescu (1999) recuerda que Lupasco (1987) demostró que la lógica del tercero incluido es verdadera, formalizable, multivalente y que no es posible contradecirla.

En el siglo $X X$ aparecieron diversas áreas que hacen parte del tercer pilar: la complejidad, y otras que pertenecían a la teoría del caos y de los procesos no-lineales, cosa que iba más allá de verificar los diferentes niveles de la realidad. Se encontró, así, que la naturaleza podría ser descrita, analizada y controlada desde términos simples correlacionados con la lógica lineal tradicional. Por el contrario, en cuanto a lo social, económico y político no había ningún avance significativo, lo cual significa que, a pesar de que la sociedad necesita adaptarse a los cambios, sigue apareciendo una lógica de simplicidad lineal en una realidad unidimensional, es decir, se continúa prevaleciendo la transmisión del conocimiento ante el desarrollo de las habilidades cognitivas del estudiante.

Por otra parte, para Edgar Morin el pensamiento complejo será lo que permite comprender la relación con un mundo y una naturaleza complejos. El propósito de este autor es el desarrollo de un pensamiento recursivo que permita conectar diferentes canales de retroalimentación en términos de conceptos como todo/parte, 
orden/desorden, observador/observado y sistema/ecosistema, para que sean tanto complementarios como antagónicos de forma simultánea (Morin, 1992). Todo lo anterior representa una reestructuración del conocimiento que, aunque parece algo utópico, toma sentido cuando es comprendido e integrado en una perspectiva nueva con respecto al mundo y junto con los otros dos pilares de la transdisciplinariedad aparece una visión más clara sobre cómo proceder.

La naturaleza y la vida en sociedad se caracterizan por tener gran cantidad de polos opuestos. En el pensamiento racional y su lógica lineal es normal separarlos. Morin califica tal separación como algo artificial ya que ni la naturaleza ni la sociedad operan con relaciones monopolares. Para contrarrestar las disfunciones que nacen entre las interrelaciones sistémicas tanto de la naturaleza y las relaciones humanas como del tejido social, se debe simplificar lo artificial y el conocimiento que se tiene sobre estos aspectos de forma ingeniosa.

Morin (1999) se basa en una amplia gama de disciplinas que él denomina "conocimiento pertinente", es decir que su visión no se limita a una disciplina y, por lo tanto, la forma como se motiva a solucionar problemas no se encuentra en el contexto de una disciplina específica. Morin no concibe lo que otros llaman una perspectiva disciplinar impulsada (Montuori, 2005). Todo lo que investiga se dirige bajo cierta demanda temática y fluctúa entre varias disciplinas que permiten obtener una perspectiva clara del conocimiento. Su visión de la transdisciplinariedad es importante al no intentar enmarcar ni totalizar de forma abstracta o enfrascarse en una sola disciplina. Su propósito es darle sentido a la vida por medio del conocimiento que llega en el proceso de búsqueda humana del cual nacen nuevos interrogantes a responder, los cuales van quedando fuera del discurso académico al ser muy complejos y abarcar una gran cantidad de disciplinas diferentes.

Morin se ha caracterizado por tener un enfoque tanto global como personal y sus trabajos posteriores se basan en la concepción de un método que no fragmente ni haga abstracta $o$ violenta la vida, que evite la unidimensionalidad, la antisepsia y la homogeneidad. Como se mencionó anteriormente, su interés se encuentra en las cuestiones existenciales, aquellas que se omiten en discursos improductivos de índole social y filosófica. En su método holográfico, resuelve asuntos políticos clave combinando la reflexión teórica y la histórica sobre el mundo a partir de ejemplos reflexivos y experienciales. Para Morín el holismo no ve más que el todo; el reduccionismo no ve más que partes, mientras que el principio hologramático inmoviliza el espíritu lineal, pues el movimiento productor del conocimiento se enriquece a través del conocimiento de las partes por el todo y el todo por las partes.

La transdisciplinariedad toma la noción de complejidad desde ciencias recientes como la física cuántica, la teoría del caos y los sistemas adaptativos complejos. Para Morin (2008) hay una diferencia entre lo complicado y lo complejo. Lo primero se refiere a aquel problema que es muy 
detallado y, por lo tanto, es difícil de resolver. Un problema complejo es aquel del cual se derivan estructuras cogerentes con patrones y ciertas características particulares, el cual nace como el producto de las relaciones interpersonales. En un problema complejo, las relaciones se mantienen adaptándose constantemente y hacen parte del núcleo del problema mismo. En estas relaciones, la información que se comparta será modificada ya que pasa de una persona a otra, hay una formación constante de energía e información que hace que el área productiva entre las disciplinas cambie constantemente. Entonces, no solo cambia dicha área sino las personas, las relaciones entre ellas, la naturaleza de la información que se comparte y los tráficos de energía, ya que para controlar y obrar en el espacio es necesario ser parte del todo (Morin y Kern, 1993). Debido a todas estas características es que resulta peculiar la resolución de un problema complejo.

\section{FORMACIÓN MULTIDIMENSIONAL}

Al momento de solucionar problemas y modelar soluciones, se considera la abstracción como una herramienta intelectual útil para la cual se necesita aplicar la lógica de forma constante. Ambos principios, lógica y abstracción, permitirán estructurar una solución más eficaz y eficientemente.

Morin (2008) menciona que un ser humano será incapaz de diferenciar o conectar un conocimiento cuando este se fragmenta, es monodisciplinar y cuantificado, porque conduce a una inteligencia ciega. Por el contrario, propone que el pensamiento complejo será el método para entender el proceso de conocimiento. Es la forma de representar el mundo y hace parte de nuestras teorías científicas, ideas y pensamientos. Él opina que la epistemología compleja es conocer el conocimiento, el cual tiene un punto de partida, pero no tiene fin; forma círculos concéntricos incansablemente, describiendo una espiral (Morin, 2004).

El pensamiento complejo tiene el objetivo de integrar las formas simplificadoras de pensar, va en contra del reduccionismo y la unidimensionalidad, de modo que, simplificar el pensamiento es desintegrar la complejidad de lo real y la complejidad no se da sobre la eliminación de la sencillez. Otro de sus objetivos es relacionar aquellas articulaciones que se han disociado en distintos dominios disciplinares, aquellos que dispersan el pensamiento (simplificación del pensar). También aspira al alcance del conocimiento multidimensional, claro está, al tener en cuenta que todavía es imposible, ya que la imposibilidad, teórica o no, de una omnisciencia, es uno de los axiomas de la complejidad (Morin, 2007).

De esta forma, se podrá utilizar la etimología del término complexus, que representa lo que está entretejido o tejido en conjunto, para explicar el concepto del pensamiento complejo. Visto de otro modo, la emergencia de las interrelaciones entre las partes y sus propiedades podrá ser de gran importancia para diferentes autores en diversas áreas del conocimiento. Se entiende que la 
emergencia son aquellas propiedades o procesos que no pueden reducirse a las propiedades o procesos de las partes que los constituyen. Esta se relaciona de forma entrañable con conceptos como la organización y el acontecer y es definida para oponerse al reduccionismo y al dualismo. La mente, por ejemplo, es uno de los fenómenos emergentes que se da a partir de la interacción distribuida entre diferentes procesos neuronales, ambientales y corporales, pero no puede ser reducida a ninguno de los componentes que hacen parte de estos.

La emergencia, epistemológicamente, se define, entonces, como la imposibilidad de un observador de pronosticar que nuevas propiedades aparezcan en un sistema. Por consiguiente, esta es una respuesta inesperada o una reacción no prevista que se da cuando las partes de un todo interactúan (Margery, 2007).

\section{CONTEXTO EDUCATIVO DESDE LA COMPLEJIDAD}

Al igual que la mente, como fenómeno emergente, el aprendizaje también surge desde una interacción distribuida entre procesos neuronales, corporales, emocionales y ambientales, y que tampoco puede reducirse a ninguno de los componentes que intervienen en él. Así, el aprendizaje, como algo complejo, será casi imposible de prever y resulta inesperado, sus contenidos pueden ser simples y complejos a la vez, pero no por ser complejo tendrá múltiples agregados de lo simple.
Moraes (2008) expresa diversos puntos de referencia para comprender el aprendizaje en entornos del constructivismo como un fenómeno emergente, imprevisible e irrepetible:

- La complejidad es dinámica y, por consiguiente, procesual. Esto implica que los procesos dinámicos sean imprevisibles y creativos, yendo más allá del horizonte de lo conocido o previsto.

- Cada una de las experiencias es única, irrepetible e intransferible, ya que el tiempo actúa en el presente sobre la materia, y el presente no puede actuar sobre el pasado. Allí recae la importancia de reconocer los momentos relevantes en la vida, tanto en lo personal como en el conocimiento y el aprendizaje.

- Para lograr una adecuada comprensión se necesita percibir la multicausalidad y multiplicidad de los fenómenos que se caracterizan por ser multidimensionales. Entender las relaciones, conexiones y enlaces es pensar de forma compleja.

- Existen múltiples realidades y al estar sujetas al observador, depende únicamente de él revelar alguna de ellas.

- Son diversas las interpretaciones de cada individuo en relación con la realidad, ya que hay muchos tipos importantes y diferentes de conocimiento.

Las capacidades cognitivas estables son desafiadas por la educación contemporánea que apoya las nuevas estructuras cognitivas, las cuales son complicadas y requieren nuevos enfoques para el proceso cognitivo. Morin (1999) presta atención en la globalización de los problemas, en que se 
vuelven transnacionales, multidimensionales transversales, transdisciplinares y planetarios. Basado en esto, afirma que la complicación es una necesidad vital para atender en un mundo donde el conocimiento debe comprenderse como un fenómeno integral. Para enfrentar los desafíos de la educación de este siglo, Morin propone el paradigma de la complejidad. Según Knyazeva (2012), la apuesta de Morin para comprender y atender estas complicaciones es el pensamiento complejo.

Knyazeva (2012) cita a Morin, para quien el pensamiento complejo es:

1. Radical y se ubica en la raíz de los problemas.

2. Multidimensional.

3. Una organización o sistema de pensamiento que analiza la relación del todo con las partes.

4. Ecológico, y aunque no aísle los objetos de estudio, considera sus interrelaciones y conexiones ecológicas en términos de autorregulación con el entorno social, natural, político, económico y cultural.

5. Un pensamiento que crea ecología de la acción y la dialéctica de la misma. Es la posibilidad de construir una estrategia con la que se pueda modificar o anular una acción que había sido emprendida por un sujeto.

6. Un pensamiento que reconoce que es imperfecto e incluyendo la duda, realiza negociaciones en la acción, ya que para realizar una acción se necesita la duda.
El pensamiento complejo debe considerarse multidimensional ya que la multidimensionalidad es una característica general de los sistemas, más que ser una herramienta para indicar determinadas características de un fenómeno descrito como conjunto; y por medio de esta noción, la intuición humana puede referirse a ciertas perspectivas amplias del mundo social y cultural. Lo anterior implica que la noción de multidimensionalidad se extiende en la filosofía social contemporánea donde hace referencia a las dimensiones humana, humanitaria, cultural y de procesos históricos. Por otra parte, este concepto está basado en la idea de autonomizar dimensiones separadas, es decir, imágenes simplificadas de fenómenos multidimensionales. Tanto en la teoría como en la práctica se deben considerar estos resultados como deformaciones profundas. Una sociedad con alternativas (multidimensional) tiene mayor oportunidad de continuar su actividad que una sin alternativas (unidimensional) en un mundo donde colapsa el fenómeno de dimensiones, lo cual demuestra que hay una inestabilidad creciente en la sociedad.

\section{CONCLUSIONES}

Tanto la sociedad como el ser humano son fenómenos multidimensionales, por lo tanto, la complejidad del ser humano se puede percibir en términos de una sociedad multidimensional. En dicha sociedad, la educación debe reconocer la multidimensionalidad de la naturaleza humana y sus múltiples niveles e insertar sus datos en ella Morin (1999). Adicionalmente, la totalidad de la sociedad está conformada por personas, 
reconocidas como piezas aisladas, pero que, al hacer parte de este todo, no deben separarse de las demás. Por ejemplo, en la economía se genera una holográmica ya que esta dimensión está en interrelación permanente con las otras dimensiones del ser humano, y en este, cosas como los deseos, las necesidades y las pasiones van más allá de los intereses propiamente económicos.

Definir una sola dimensión como lo absoluto es lo que ha llevado al ser humano a tener una visión tergiversada. Por ejemplo, al nombrar a los seres humanos como homo sapiens, se ha difundido el racionalismo en las investigaciones antropológicas y se ha afirmado el concepto del antropocentrismo.

Por este motivo es que la educación debe promover que los individuos se adapten a un medio de constante cambio y de condiciones multidimensionales, por medio de la búsqueda de estrategias, modelos de comportamiento y nuevos puntos de vista que correspondan a las características de las nuevas generaciones. Morin también menciona la urgencia de desfragmentar el conocimiento, ya que su dispersión evita que la complejidad y diversidad de los seres humanos y su entorno se aclare. De acuerdo con Lyotard (2001), el desarrollo del conocimiento científico actual da como resultado este problema porque se ponen en duda las líneas que dividen las diferentes áreas de la ciencia $\mathrm{y}$, por consiguiente, desaparecen las disciplinas; al superponerse estos nuevos espacios, nacen fronteras entre las ciencias.
Bajo este contexto nace el conocimiento en el proceso de formación como parte de los aprendices, de sus intereses, los valores que los caracterizan y las metas por cumplir. Así se entiende que la naturaleza del conocimiento está supeditada al individuo. La educación debe propender por el desarrollo de habilidades y capacidades vitales en las personas, por ello, está directamente relacionada con su mundo a través de una práctica tan cotidiana como lo es la participación.

La forma práctica de abordar los problemas sistemáticamente es lo que define la transdisciplinariedad, pero todavía se considera un proyecto incompleto que necesita de la ejecución de muchas sistematizaciones. La disciplina hace referencia a un solo nivel de la realidad mientras que la transdisciplina se extiende de forma transversal en varios niveles. Ambas son complementarias, pero pasar de una a otra está en el recorrido por diferentes niveles de la realidad que permite enriquecerlos recíprocamente y facilita comprender la complejidad.

La transdisciplinariedad es una nueva perspectiva, más sistémica y global sobre el mundo. A pesar de entenderse sus posibles aplicaciones como metodología en las ciencias e ingenierías, la transdisciplinariedad todavía posee deficiencias. Se necesita una claridad mayor en cuanto a los niveles de la realidad y relacionarlos con las problemáticas del mundo tales como el biocentrismo y el antropocentrismo, el desarrollo y el medio ambiente, en relación con los anteriores y 
en su función de opuestos o complementarios, los conceptos de razón e intuición, entre otros.

Dichas problemáticas representarán cuestiones abiertas para ser discutidas en entornos como el educativo donde la idea de mejorar la compresión del mundo y la naturaleza sean las bases para consolidar la transdisciplinariedad como proyecto. Serna (2015) manifiesta que, si no se lleva a cabo este esfuerzo, crecerán los daños a la sociedad y a la naturaleza ya que las acciones y suposiciones sobre su comprensión se encuentran fragmentadas, limitadas y vistas de forma subjetiva o parcial. El reto es perfeccionar la transdisciplinariedad como una perspectiva del mundo al practicarla sistemáticamente $\mathrm{y}$ considerando las posibilidades.

Cabe destacar que en la actualidad no se conocen universidades orientadas a formar a sus estudiantes de forma transdisciplinar, por lo tanto, es necesario definir espacios para aplicarla y desarrollarla. La necesidad de este tipo de formación es algo urgente para los sistemas educativos actuales. Se requiere un nuevo sistema formatico que forme profesionales capaces de lograr los objetivos verticales y horizontales del curso por medio de proyectos que sean integradores, multidimensionales y transdisciplinares.

La forma de adquirir los conocimientos está cambiando. El pensamiento complejo en lugar de ser una forma definida, es una manera de adquirirlos. La educación debe estimular el potencial interno de cada individuo ya que la configuración de sus conocimientos dependerá de su reflexión, más allá de encontrar un simple significado. Los métodos conectados con la naturaleza interna del ser humano deben utilizarse y potenciarse con el fin de ampliar las posibilidades del ser por medio de la exploración de su naturaleza multidimensional, siendo este un homo complexus. Algunos de estos métodos son el juego, el diálogo, la investigación, entre otros. Así pues, el plan de estudios deberá permitir este tipo de interacciones de la meta-comunicación y todo tipo de interacciones educativas, pero basado en:

- La transfenomenalidad, que solo es posible al considerar simultáneamente los factores y eventos asociados con los distintos niveles en los que se pueden explicar los fenómenos. En el paradigma de la complejidad, se ofrece como un enfoque para la actividad cognitiva y se define como un atributo del entorno educativo y del ser humano.

- La transdisciplinariedad, que supone la consideración simultánea de los eventos que tienen relación con las diversas perspectivas disciplinares lo cual la convierte en una medida del conocimiento científico.

- La transdiscursividad, en la que se consideran simultáneamente aquellos hechos que se relacionan con varias perspectivas discursivas, cosa que la define como una práctica comunicativa y a su vez, cognitiva.

- Por último, la transculturalidad, que solo se da cuando al considerar simultáneamente los hechos de diferentes tradiciones y orientaciones de los valores culturales. En el paradigma de la complejidad, es ofrecida como una situación en la cultura bajo el contexto de las ideas. 


\section{REFERENCIAS}

Goorhuis, H. (2000). Second order management for emerging problems. En Häberli, R. et al. (eds.), Transdisciplinarity: Joint problemsolving among science (pp. 25-29). Zúrich: Haffmans Sachbuch Verlag.

Häberli, R., Bill, A., Grossenbacher-Mansuy, W., Klein, J., Scholz, R. y Welti, M. (eds.). (2001). Transdisciplinarity: Joint problemsolving among science, technology and society. Basilea: Birkhäuser Verlag.

Häberli, R., Bill, A., Grossenbacher-Mansuy, W., Klein, J., Scholz, R. y Welti, M. (2001a). Synthesis. En Häberli, R. et al. (eds.), Transdisciplinarity: Joint Problem Solving Among Science, Technology, and Society, 622. Doi: 10.1007/978-3-0348-8419-8_2

Knyazeva, H. (2012). Complex Thinking: Methodological, managerial and ethical aspects. En Symposium Physical and Metaphysical Aspects of Systems after Morin (pp. 47-50). Simposio llevado a cabo por el Bertalanffy Center for the Study of Systems Science, Viena, Austria.

Lupasco, S. (1987). Le principe d'antagonisme et la logique de l'énergie. París: Éditions du Rocher.

Lyotard, J. (2001). The postmodern condition Continental Philosophy. En Seidman, S. y Alexander, J. (eds.), The New Social Theory Reader - Contemporary Debates (pp. 166176). Londres: Routledge.

Maldonado, C. (2007). Complejidad: ciencia, pensamiento y aplicaciones. Bogotá: Universidad Externado de Colombia.

Margery, E. (2007). Las Organizaciones del Siglo XXI. En Conversatorios sobre Pensamiento Complejo. Comunicación oral llevada a cabo en la Universidad de Costa Rica, San José, Costa Rica.

Montuori, A. (2005). Gregory Bateson and the challenge of transdisciplinarity. Cybernetics and Human Knowing, 12(1-2), pp. 147-158.
Moraes, M. (2008). Dimensoes metodológicas e suas implicacoes educacionais. Taguatinga: Universidad Católica de Brasilia.

Morin, E. (1992). From the concept of system to the paradigm of complexity. Journal of Social and Evolutionary Systems, 15(4), 371-385. Doi: 10.1016/1061-7361(92)90024-8

Morin, E. y Kern, A. (1993). Tierra-patria. Barcelona: Kairós.

Morin, E. (1999). Los siete saberes necesarios para la educación del futuro. París: Santillana.

Morin, E. (2004). La Méthode 6 - Éthique. París: Seuil.

Morin, E. (2007). Complexité restreinte, complexité générale. En Le Moigne, J. y Morin, M. (eds.), Intelligence de la complexité Epistémologie et pragmatique (pp. 28-50). La Tour-d'Aigues: Éditions de l'Aube.

Morin, E. (2008). On complexity. Nueva Jersey: Hampton Press.

Nicolescu, B. (1996). La transdisciplinarité : Manifeste. París: Éditions du Rocher.

Nicolescu, B. (1999). Stéphane Lupasco, l'homme et l'œuvre. Mónaco: Éditions du Rocher.

Nicolescu, B. (2000). Transdisciplinarity and complexity: Levels of reality as source of indeterminacy. En Arecchi, T. (ed.), Determinismo e complessità (pp. 127-142). Roma: Armando Editore.

Serna, E. (2015). Por qué falla el sistema de educación (1. ${ }^{\mathrm{a}}$ ed.). Medellín: Editorial IAI. 